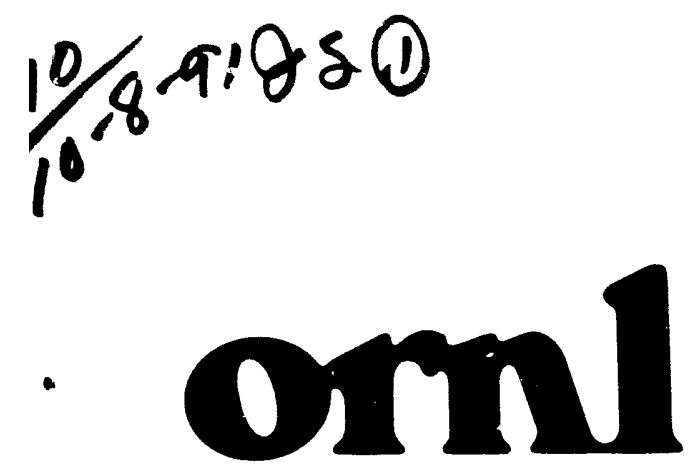

OAK RIDGE

NATIONAL

LABORATORY

MARTIN MARIETRAa
ORNL/TM-11895

\title{
A Standard Test Set for Numerical Approximations to the Shallow Water Equations in Spherical Geometry
}

\author{
David L. Williamson \\ John B. Drake \\ James J. Hack \\ Rüdiger Jakob \\ Paul N. Swarztrauber
}


This report has ben reproduced directly from the best available copy

Available to DOE and DOE contractors from the Office of Scientific and Technical Information, P.O. Box 62, Oak Ridge, TN 37831; prices available from (615) 576-8401, FTS 626-8401.

Available to the public from the National Tochnical Information Service, U.S. Department of Commerce, 5285 Port Royal Rd., Springfield, VA 22161.

This report was prepared as an account of work sponsored by an agency of the United States Government. Neither the United States Government nor any agency thereof, nor any of their employees, makes any warranty, express or implied, or assumes any legal liability or responsibility for the accuracy, completeness, or usefulness of any information, apparatus, product, or process disclosed, or represents that its use would not infringe privately owned rights. Reference herein to any specific commercial product, process, or service by trade name, tredemark, manufacturer, or otherwise, does not necessarily constitute or imply its endorsement, recommendation, or favoring by the United States Government or any agency thereof. The viev's and opinions of authors expressed herein do not necessarily state or reflect those of the United States Government or any agency thereof. 
O ORNL/TM--11895

Engineering Physics and Mathematics Division

DE92 000385

Mathematical Sciences Section

\section{A STANDARD TEST SET FOR NUMERICAL APPROXIMATIONS TO THE SHALLOW WATER EQUATIONS IN SPHERICAL GEOMETRY}

David L. Williamson $\dagger$

John B. Drake $\ddagger$

James J. Hack ${ }^{\dagger}$

R üdiger Jakob $\dagger$

Paul N. Swarztrauber $\dagger$

$\dagger$ National ('enter for Atmospheric Research

Table Mesa Drive

Boulder, CO 80:307

\#athematical Sciences Section

Oak Ridge National Laboratory

P.O. Box 2008, Bldg. 6012

Oak Ridge, TN $37831-63667$

Date Published: August 1991

Research was struported by the CHAMMP Program of the Office of IIcalth and Environmental Research, U.S. Department of Energy.

Prepared by the

Oak Ricke National Laboratory

()ak Rirge. T'ennessee 37s:31

mallaged by

Martin Narienta Energy Sistems. Inc.

for the

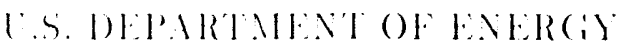

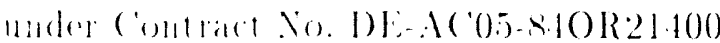




\section{Contents}

1 Introduction . . . . . . . . . . . . . . . . . . . . 1

2 The Shallow Water Equations on a Sphere . . . . . . . . . . . . 3

2.1 Flux Form . . . . . . . . . . . . . . . . . . . 3

2.2 Advective Form . . . . . . . . . . . . . . . . 4

2.3 Vorticity Divergence Form . . . . . . . . . . . . . . . . 4

2.4 Bounded Differential Expression Form . . . . . . . . . . . . 6

2.5 Stream Function, Velocity Potential Form . . . . . . . . . . . 6

2.6 General Orthogonal Coordinates . . . . . . . . . . . . 7

2.7 Three-Dimensional, Constrained Form . . . . . . . . . . 8

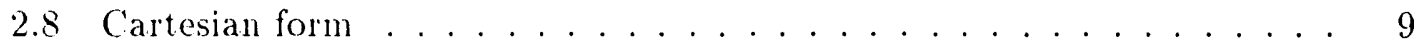

3 Test Cases . . . . . . . . . . . . . . . . . . . . . . 10

3.1 Advection of Cosine Bell over the Pole . . . . . . . . . . . . . . . 11

3.2 Global Steady State Nonlinear Zonal Geostrophic Flow . . . . . . . . . 12

3.3 Steady State Nonlinear Zonal (ieostrophic Flow with Compact Support 14

3.4 Forced Nonlinear System with a Translating Low . . . . . . . . . . . . . 16

3.5 Zonal Flow Over an Isolated Mountain . . . . . . . . . . . . . . 18

3.6 Rossby-Haurwitz Wave . . . . . . . . . . . . . . . . . 18

3.7 Analyzed $500 \mathrm{mb}$ Height and Wind Field Initial Conditions . . . . . 20

4 Performance Benchmark . . . . . . . . . . . . . . . . . . 20

5 General Comments . . . . . . . . . . . . . . . . . 21

6 References . . . . . . . . . . . . . . . . . . . 22 


\section{Acknowledgements}

We would like to thank many of our colleagues for discussions and encouragement related to these test cases. Although we cannot mention everyone we would like to thank in particular J. Dukowicz and J. Côté for detailed comments which led us to modify our original test cases and error measures. This report is one of a series concerning the use of numerical methods for global climate modeling. The work reported is sponsored by the CHAMMP initiative of the Atmospheric and Climate Research Division of the Office of Energy Research, U.S. Department of Energy. The National Center Eor Atmospheric Research is sponsored by the National Science Foundation. 


\title{
A STANDARD TEST SET FOR NUMERICAL APPROXIMATIONS TO THE SHALLOW WATER EQUATIONS IN SPHERICAL GEOMETRY
}

\author{
David L. Williamson \\ John B. Drake \\ James J. Hack \\ Rüdiger Jakob \\ Paul N. Swarztrauber
}

\begin{abstract}
A suite of seven test cases is proposed for the evaluation of numerical methods intended for the solution of the shallow water equations in spherical geometry. The shallow water equations exhibit the major difficulties associated with the horizontal dynamical aspects of atmospheric modeling on the spherical earth. These cases are designed for use in the evaluation of numerical methods proposed for climate modeling and to identify the potential trade-offs which must always be made in numerical modeling. Before a proposed scheme is applied to a full baroclinic atmospheric model it must perform well on these problems in comparison with other currently accepted numerical methods. The cases are presented in order of complexity. They consist of advection across the poles, steady state geostrophically balanced flow of both global and local scales, forced nonlinear advection of an isolated low, zonal flow impinging on an isolated mountain, Rossby-Haurwitz waves and observed atmospheric states. One of the cases is also identified as a computer performance/algorithm efficiency benchmark for assessing the performance of algorithms adapted to massively parallel computers.
\end{abstract}




\section{Introduction}

The early days of global atmospheric modeling saw significant efforts in adapting then current numerical methods to solving fluid flow on the surface of the sphere. A large component of this effort was directed toward finite difference approaches. The review article by Williamson [28] discusses the many finite difference approaches that were applied to the problem at that time and gives a lengthy list of references. The introduction of the spectral transform method by Orszag [15], and Eliasen, Machenhauer and Rasmussen [7] made the spectral method cost effective in terms of storage and processor time compared with finite difference approaches. The review by Machenhauer [13] discusses the various applications of the spectral method in detail. The spectral method presents a natural solution to problems introduced by spherical geometry in part because it provides an isotropic representation in spectral space even though the commonly adopted underlying Gaussian grid does not. The spectral transform method is widely accepted as the basis of operational numerical weather prediction and global climate models. Although not universally adopted the method has become the rule rather than the exception. As a result little effort has been directed in the last decade toward developing alternative methods of approximation for global atmospheric models.

Currently there is renewed interest in alternative methods for a variety of reasons. The European Centre for Medium Range Weather Forecasts (ECMWF) has reported [6] that at resolutions greater than those currently used in in operational numerical forecast models the computational cost of the Legendre transform associated with the spectral method will become a significant fraction of the total cost of the model. Thus other methods are likely to become economically competitive. The spectral representation contributes to unphysical structures in the pres ted fields such as negative water vapor $[20]$. Traditional finite difference approximations also suffer from this defect. However, recently shape preserving and essentially non-oscillatory schemes have been developed to address this deficiency. Spectral models require a global domain and have thus been based on a normalized vertical coordinate such as pressure divided by surface pressure. Over steep mountains the horizontal pressure gradient force in such systems is a small difference of two large terms and difficult to approximate accurately. Mesh refinement near mountains, or admittance of explicit lateral boundaries where mountains can penetrate the grid, appear as potential alternatives. The spectral method also presents problems with efficient implementation on some of the new computer architectures althongh these are not necessarily unique to the spectral method. The global communication required by the spectral transform may be difficult to achieve efficiently on massively parallel computers with distributed memory. With grid point based schemes a similar communication problem may arise however associated with the elliptic problem introduced by a semi-implicit time stepping algorithm.

The renewed interest in algorithm development has led to the need to define standard test cases with which potential schemes may be compared. Strict comparisons based on such test cases will aid in rationally choosing the compromises which must be made in mumerical modeling. We present a suite of test cases in this report for numerical approximations to the shallow water equations in spherical geonetry. The shallow water equations on a rotating sphere serve as a primaly test problem for numerical 
methods used in modeling global atmospheric flows. They describe the behavior of a shallow homogeneous incompressible and inviscid fluid layer. They present the major difficulties found in the horizontal aspects of three dimensional global atmospheric modeling. Thus they provide a first test to wced out potentially non-competitive schemes without the effort of building a complete model. However, because they do not represent the complete atmospheric system, the shallow water equations are only a first test. Ultimately schemes which look attractive based on these tests must be applied to the complete baroclinic problem. We hope that the existence of a standard test set for the shallow water equations will encourage the continued exploration of alternative numerical methods and provide the community with a mechanism for judging the relative merits of numerical schemes and parallel computers for atmospheric flow calculations.

We present here a suite of seven test cases in increasing order of complexity. Several analytic treatments included in the suite provide objective standards for judging the accuracy of numerical schemes and provide quick checks on the validity of code. The first test consists of advection of a structure of compact support by a specified wind field corresponding to solid body rotation whose axis is not necessarily coincident with that of the rotation of the earth. As such this case deals with only a subset of the shallow water equations, namely the continuity equation, but concentrates on a scheme's ability to deal with the poles of the spherical coordinate system.

The second and third cases present steady state, nonlinear zonal geostrophic flow. They are a global form with the wind corresponding to solid body rotation and a local form where the wind field has compact support. In both cases the spherical coordinate poles are not necessarily coincident with the earth's rotation axis. As with the first case these test a scheme's ability to handle the poles, but in addition nonlinearities can come into play.

The succeding test cases are of increasing complexity and realism, exercising the more subtle aspects of atmospheric flows. One case uses an analytic forcing function to drive a low around the sphere. The case mimics the more complicated local structures observed in the atmosphere. Another case consists of zonal flow impinging on an isolated mountain in which a downstream wavetrain is set up. A Rossby-Haurwitz wave case is also included. Analytic solutions for the Rossby-Haurwitz wave in the shallow water context are not known but this wave has become a standard test case in meteorology. A reference solution is provided by a high resolution spectral transform model integration. Finally, actual weather patterns are presented for initial conditions. Since they obviously have no analytic solution a reference solution is provided again by a high resolution spectral transform model run. As mentioned above, analytic solutions for the last three cases are not known. Reference solutions will be provided by a high resolution spectral transform model. For it to be accepted it must be duplicated by a high resolution solution provided hy at least one other different method.

With rach test case we ask for a rariety of specifie measures of the error of the munerical solution. Just an there in no single ideal test case, there is no single measure that determines the quality of a scheme for atmospheric modeling. We include the variety of test casen and error meanures to provide as much information as possible to would-be users so they can evaluate the various tradeoffs involved with the schemes.

The second test in the suite is atso prepesed as a performance benchmarking prob. 
lem. Such benchmarking is particularly important since the efficiency of schemes must be evaluated considering the computing environment for which they are designed.

As an initial basis of comparison we provide in a companion report solutions to these problems from a spectral transform approximation at resolutions currently used in atmospheric nodels. Spectral models are widely but not universally adopted in climate modeling and numerical weather predicition. We encourage centers currently using other method's to run these tests with their schemes and to submit the results for comparison. To facilitate comparison of schemes, a machine readable copy of standard FORTRAN routines which calculate the initial conditions and analytic or reference solutions is available from netlib@ornl.gov. A file summarizing performance statistics contributed by members of the community will also be maintained. In addition a list of corrections to this paper will be maintained along with a bibliography of reports presenting results of tests of numerical schemes based on this test suite and any modifications to the test suite generally agreed upon by the community. Please submit additionai performance data and references for the bibliography as they become available to John Drake (bbdGiornl.gov).

\section{The Shallow Water Equations on a Sphere}

For convenience, we summarize many forms in which the shallow water equations can be written. The reader is referred to standard texts such as Holton [10] and Haltiner and Williams [8] for more general development.

\subsection{Flux Form}

The shallow water equations on a rotating sphere can be written in flux form as

$$
\frac{\partial h^{*} \mathbf{v}}{\partial t}+\nabla \cdot\left(\mathbf{v} h^{*} \mathbf{v}\right)=-f \hat{\mathbf{k}} \times h^{*} \mathbf{v}-g h^{*} \nabla h
$$

and

$$
\frac{\partial h^{*}}{\partial t}+\nabla \cdot\left(h^{*} \mathbf{v}\right)=0
$$

where $h^{*}$ is the depth of the fluid and $h$ is the height of the free surface above a reference sphere (sea level). If $h_{s}$ denotes the height of the underlying mountains, $h=h^{*}+h_{s}$. The horizontal (on the sphere) vector velocity is denoted $\mathrm{v}$ with components $u$ and $v$ in the longitudinal $(\lambda)$ and latitudinal $(\theta)$ directions respectively. The $\nabla$ operator is the spherical horizontal gradient operator given by

$$
\Gamma() \equiv \frac{\hat{\mathrm{i}}}{a \cos \theta} \frac{\partial}{\partial \lambda}()+\frac{\hat{\mathrm{j}}}{a} \frac{\partial}{\partial \theta}()
$$

and $\nabla$. is the spherical horizontal divergence operator given by

$$
\Gamma \cdot \mathrm{v} \equiv \frac{1}{u \cos \theta}\left[\frac{\partial u}{\partial \lambda}+\frac{\partial(v \cos \theta)}{\partial \theta}\right]
$$


The longitudinal, latitudinal and outward radial unit vectors are $\hat{\mathbf{i}}, \hat{\mathbf{j}}$ and $\hat{\mathbf{k}}$, respectively, $f$ is the Coriolis parameter. $g$ is the gravitational constant and $a$ is the radius of the earth. The Coriolis parameter is given by $2 \Omega \sin \theta$ where $\Omega$ is the rotation rate of the earth.

The equations for the spherical components can be derived by writing $\mathbf{v}=u \hat{\mathbf{i}}+v \hat{\mathbf{j}}$ and using

$$
\frac{d h^{*} \mathbf{v}}{d t}=\hat{\mathrm{i}} \frac{d h^{*} u}{d t}+\hat{\mathbf{j}} \frac{d h^{*} v}{d t}+h^{*} u \frac{d \hat{\mathbf{i}}}{d t}+h^{*} v \frac{d \hat{\mathbf{j}}}{d t} .
$$

Equation (1) in terms of spherical components is then

$$
\begin{gathered}
\frac{\partial h^{*} u}{\partial t}+\nabla \cdot\left(h^{*} u \mathbf{v}\right)-\left(f+\frac{u}{a} \tan \theta\right) h^{*} v+\frac{g h^{*}}{a \cos \theta} \frac{\partial h}{\partial \lambda}=0 \\
\frac{\partial h^{*} v}{\partial t}+\nabla \cdot\left(h^{*} v \mathbf{v}\right)+\left(f+\frac{u}{a} \tan \theta\right) h^{*} u+\frac{g h^{*}}{a} \frac{\partial h}{\partial \theta}=0 .
\end{gathered}
$$

\subsection{Advective Form}

The advective form of the horizontal momentum and mass continuity equations can be written

$$
\frac{d \mathbf{v}}{d t}=-f \hat{\mathbf{k}} \times \mathbf{v}-g \nabla h .
$$

and

$$
\frac{d h^{*}}{d t}+h^{*} \nabla \cdot \mathbf{v}=0
$$

where the substantial derivative is given by

$$
\frac{d}{d t}() \equiv \frac{\partial}{\partial t}()+(\mathbf{v} \cdot \nabla)() \text {. }
$$

The equations for the spherical components are

$$
\begin{gathered}
\frac{\partial u}{\partial t}+\mathrm{v} \cdot \nabla u-\left(f+\frac{u}{a} \tan \theta\right) v+\frac{g}{a \cos \theta} \frac{\partial h}{\partial \lambda}=0, \\
\frac{\partial v}{\partial t}+\mathrm{v} \cdot \nabla v+\left(f+\frac{u}{a} \tan \theta\right) u+\frac{g}{a} \frac{\partial h}{\partial \theta}=0,
\end{gathered}
$$

and

$$
\frac{\partial h^{*}}{\partial t}+\mathbf{v} \cdot \nabla h^{*}+\frac{h^{*}}{a \cos \theta}\left(\frac{\partial u}{\partial \lambda}+\frac{\partial v \cos \theta}{\partial \theta}\right)=0 .
$$

\subsection{Vorticity Divergence Form}

The horizoital momentum can also be specified in terms of relative vorticity,

$$
\dot{i} \equiv \mathrm{k} \cdot(\Gamma, \mathrm{v}) \text {. }
$$

and horizonial divergence.

$$
r \equiv \Gamma \cdot \mathbf{v}
$$


The curl operator is given by

$$
\hat{\mathbf{k}} \cdot(\nabla \times \mathbf{v})=\frac{1}{a \cos \theta}\left[\frac{\partial v}{\partial \lambda}-\frac{\partial u \cos \theta}{\partial \theta}\right] .
$$

Using the vector identity

$$
(\mathbf{v} \cdot \nabla) \mathbf{v}=\nabla\left(\frac{\mathbf{v} \cdot \mathbf{v}}{2}\right)+\zeta \hat{\mathbf{k}} \times \mathbf{v}
$$

the horizontal momentum equation becomes

$$
\frac{\partial \mathbf{v}}{\partial \mathbf{t}}=-(\zeta+f) \hat{\mathbf{k}} \times \mathbf{v}-\nabla\left(g h+\frac{\mathbf{v} \cdot \mathbf{v}}{2}\right),
$$

or in spherical component form

$$
\begin{gathered}
\frac{\partial u}{\partial t}=(\zeta+f) v-\frac{1}{a \cos \theta} \frac{\partial}{\partial \lambda}\left[g h+\frac{1}{2}\left(u^{2}+v^{2}\right)\right] \\
\frac{\partial v}{\partial t}=-(\zeta+f) u-\frac{1}{a} \frac{\partial}{\partial \theta}\left[g h+\frac{1}{2}\left(u^{2}+v^{2}\right)\right] .
\end{gathered}
$$

Applying the curl and divergence operators $\hat{\mathrm{k}} \cdot \nabla \times()$ and $\nabla \cdot()$ to the momentum equation yields

$$
\begin{gathered}
\frac{\partial \zeta}{\partial t}=-\nabla \cdot(\zeta+f) \mathbf{v} \\
\frac{\partial \delta}{\partial t}=\hat{\mathbf{k}} \cdot \nabla \times(\zeta+f) \mathbf{v}-\nabla^{2}\left(g h+\frac{\mathbf{v} \cdot \mathbf{v}}{2}\right),
\end{gathered}
$$

or in terms of spherical components

$$
\begin{aligned}
\frac{\partial \dot{\zeta}}{\partial t}= & -\frac{1}{a \cos \theta} \frac{\partial}{\partial \lambda}[(\zeta+f) u] \\
& -\frac{1}{a \cos \theta} \frac{\partial}{\partial \theta}[(\zeta+f) v \cos \theta] \\
\frac{\partial \delta}{\partial t}= & \frac{1}{a \cos \theta} \frac{\partial}{\partial \lambda}[(\zeta+f) v] \\
& -\frac{1}{a \cos \theta} \frac{\partial}{\partial \theta}[(\zeta+f) u \cos \theta] \\
& -\Gamma^{2}\left[g h+\frac{1}{2}\left(u^{2}+v^{2}\right)\right]
\end{aligned}
$$

where

$$
\nabla^{2}()=\frac{1}{a^{2} \cos ^{2} \theta} \frac{\partial^{2}()}{\partial \lambda^{2}}+\frac{1}{a^{2} \cos \theta} \frac{\partial}{\partial \theta}\left(\frac{\cos \theta \partial()}{\partial \theta}\right)
$$




\subsection{Bounded Differential Expression Form}

The spherical vector component forms of the equations contain individual unbounded differential expressions approaching the poles. Swarztrauber [3] has developed a form for the equations containing only bounded differential expressions

$$
\begin{aligned}
& \frac{\partial u}{\partial t}=-\frac{u}{a} \delta-\frac{v}{a} \frac{\partial u}{\partial \theta}+\frac{u}{a} \frac{\partial v}{\partial \theta}+f v-\frac{g}{a \cos \theta} \frac{\partial h}{\partial \lambda} \\
& \frac{\partial v}{\partial t}=-\frac{u}{a} \zeta-\frac{u}{a} \frac{\partial u}{\partial \theta}-\frac{v}{a} \frac{\partial v}{\partial \theta}-f u-\frac{g}{a} \frac{\partial h}{\partial \theta} .
\end{aligned}
$$

\subsection{Stream Function, Velocity Potential Form}

The spherical velocity components can be avoided by the introduction of a horizontal stream function, $\iota "$, and velocity potential, $x$. The equation relating horizontal velocity and these two scalar quantities is

$$
\mathrm{v}=\hat{\mathrm{k}} \times \nabla \psi+\nabla \lambda
$$

The spherical wind components are related to the stream function and velocity potential by

$$
\begin{gathered}
u=-\frac{1}{a} \frac{\partial \psi}{\partial \theta}+\frac{1}{a \cos \theta} \frac{\partial \chi}{\partial \lambda} \\
v=\frac{1}{a \cos \theta} \frac{\partial \psi}{\partial \lambda}+\frac{1}{a} \frac{\partial \lambda}{\partial \theta} .
\end{gathered}
$$

The application of the curl and divergence operators to (29) and (30) gives the absolute vorticity

$$
\eta \equiv \zeta+f=\Gamma^{2} \psi+f
$$

and divergence

$$
\delta=\nabla^{2} \lambda
$$

In terms of the strean function and velocity potential the horizontal momentum and mass continuity equations can be written [1-4]

$$
\begin{gathered}
\frac{\partial \eta}{\partial t}+\Gamma \cdot(\eta \Gamma \backslash)-J(\eta, \psi)=0, \\
\frac{\partial \delta}{\partial t}-\Gamma \cdot(\eta \Gamma \varphi)-J(\eta, \lambda)=-\nabla^{2}(\Lambda+g h),
\end{gathered}
$$

and

$$
\frac{\partial h^{*}}{\partial t}+\Gamma \cdot\left(h^{*} \Gamma \lambda\right)-J\left(h^{*} \cdot \psi\right)=0 .
$$

In spherical coordinates the Jacobian operator is defined by

$$
J(a, 3)=\frac{1}{a^{2} \cos ^{2} \theta}\left(\frac{\partial a}{\partial \lambda} \frac{\partial \beta}{\partial \theta}-\frac{\partial a}{\partial \theta} \frac{\partial \beta}{\partial \lambda}\right) .
$$

Here $K$ is the kinetic energ! $\frac{1}{2}\left(u^{2}+r^{2}\right)$ and can be expressed in terms of stream function 
and velocity potential as

$$
\left.K=\frac{1}{2}\left[\Gamma \cdot(\iota \cdot \Gamma \iota)-\iota \cdot \Gamma^{2} \psi+\Gamma \cdot(\lambda \Gamma\rangle\right)-\lambda \nabla^{2} \chi\right]+J(\psi, \chi) .
$$

The $J$ an $1 \Gamma$. operators, curl and divergence, have the following integral properties according to Gauss's theorem.

$$
\iint_{A} J(\alpha, \beta) d A=\int_{C} \alpha \frac{\partial \beta}{\partial s} d s
$$

and

$$
\iint_{A} \Gamma \cdot(\alpha \Gamma \beta) d A=\int_{C} a \frac{\partial \beta}{\partial n} d s
$$

where $\frac{\partial}{\partial s}$ is the derivative along $C^{\prime}$ and $\frac{\partial}{\partial n}$ is the derivative normal to the curve.

\subsection{General Orthogonal Coordinates}

The general orthogonal coordinate form is useful when considering approximations based on various map projections. Let $(x, y)$ be the orthogonal coordinates and $m_{x}$ and $m_{y}$ be the metric coefficients so the distance increment $(d \ell)$ satisfies

$$
(d 6)^{2}=m_{x}^{2} d x^{2}+m_{y}^{2} d y^{2} .
$$

The velocity vector $v$ has components

$$
\begin{aligned}
& l=m_{x} \cdot \frac{d x}{d t} \\
& I=m_{y} \frac{d y}{d t}
\end{aligned}
$$

in the $x$ and $y$ directions. respectively. The equations of motion are

$$
\begin{aligned}
& \frac{d l}{d t}-\left[f+\frac{1}{m_{x} m_{y}}\left(1 \cdot \frac{\partial m_{y}}{\partial x}-l \cdot \frac{\partial m_{x}}{\partial y}\right)\right] \downarrow+\frac{g}{m_{x}} \frac{\partial h}{\partial x}=0 \\
& \frac{d l}{d t}+\left[f+\frac{1}{m_{x} m_{3}}\left(1 \cdot \frac{\partial m_{y}}{\partial x}-l \cdot \frac{\partial m_{x}}{\partial y}\right)\right] \zeta+\frac{g}{m_{y}} \frac{\partial h}{\partial y}=0
\end{aligned}
$$

where

$$
\frac{d}{d t}=\frac{\partial}{\partial t}+\frac{l}{m_{z}} \frac{\partial}{\partial x}+\frac{1}{m_{y}} \frac{\partial}{\partial y}
$$

The continuity equation in

$$
\frac{d h^{*}}{d t}+\frac{h^{*}}{m_{x} m_{i}}\left[\frac{\partial}{\partial I^{\prime}}\left(m_{y}()+\frac{\partial}{d y}\left(m_{x} \cdot\right)\right]=0\right.
$$


Note, for spherical coordinates

$$
\begin{gathered}
x=\lambda, \quad y=\theta \\
m_{x}=a \cos \theta, \quad m_{y}=a \\
u=m_{x} \frac{d x}{d t}=a \cos \theta \frac{d \lambda}{d t} \\
v=m_{y} \frac{d y}{d t}=a \frac{d \theta}{d t} .
\end{gathered}
$$

Commonly used map projections are north and south polar stereographic

$$
m_{x}=m_{y}=\frac{1}{2}(1 \pm \sin \theta)=\frac{4 a^{2}}{x^{2}+y^{2}+4 a^{2}}
$$

and Mercator"s

$$
m_{x}=m_{y}=\cos \theta .
$$

Al! ma jor map projections are described from a geographical point of view by Steers [23]. Saucier [22] discusses the common projections used in meteorology. More recently Pearson [17] has summarized the field.

\subsection{Three-Dimensional, Constrained Form}

('oté [4] developed a three-dinensional vector form for the horizontal momentum equations using the undetermined Lagrange multiplier method to constrain the motion to be on the surface of the sphere.

$$
\frac{d \mathrm{~V}}{d t}=\mathbf{F}+\mu \mathbf{r}
$$

where

$$
\mathrm{V}=\frac{d \mathbf{r}}{d t}
$$

is the three-dimensional velocity vector in a rotating frame.

$$
\mathbf{F}=-f \mathbf{r} \times \mathbf{V}-g \Gamma h
$$

and $\mu$ is the lagrange multipler determined by requiring

$$
\mathbf{r} \cdot \mathbf{r}=a^{2}
$$

be satisfied for all time: $\mathrm{r}$ is the position vector. Evaluation of the Lagrange multiplier for the continuous equations given $\mu=-\mathrm{V} \cdot \mathrm{V}$ and leads to the usual Eulerian form. There are advantages. howerer. in determining the Lagrange multiplier after the time discretization [5]. In this approach the thees-dimensional equation is solved rather than

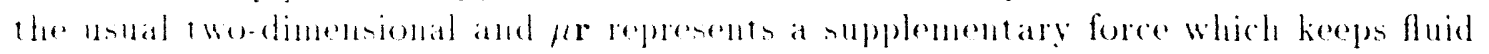

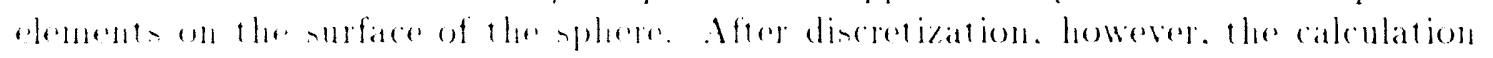
can be carried out in two-dimemintal-pate. 


\subsection{Cartesian form}

It may be advantageous to evaluate the surface derivatives using a Cartesian form. By extending the surface vector $\mathbf{v}=(u, v)^{T}$ to the three-dimensional $\mathbf{v}_{s}=(w, v, u)^{T}$ the shallow water equations can be embedded in the system

$$
\frac{\partial \mathbf{v}_{s}}{\partial t}+\mathbf{S}\left(\mathbf{v}_{s}\right) \mathbf{v}_{s}+\alpha+\beta+\delta=0
$$

where

$$
\mathbf{S}\left(\mathbf{v}_{s}\right)=\left(\begin{array}{ccc}
\frac{\partial w}{\partial r} & \frac{1}{a}\left(\frac{\partial w}{\partial \theta}-v\right) & \frac{1}{a \cos \theta}\left(\frac{\partial w}{\partial \lambda}-u \cos \theta\right) \\
\frac{\partial v}{\partial r} & \frac{1}{a}\left(\frac{\partial v}{\partial \theta}+w\right) & \frac{1}{a \cos \theta}\left(\frac{\partial v}{\partial \lambda}-u \sin \theta\right) \\
\frac{\partial u}{\partial r} & \frac{1}{a} \frac{\partial u}{\partial \theta} & \frac{1}{a \cos \theta}\left(\frac{\partial u}{\partial \lambda}-v \sin \theta+w \cos \theta\right)
\end{array}\right),
$$

$r$ is the radial coordinate ( $r=a$ at the earth's surface) and

$$
\begin{gathered}
\boldsymbol{\alpha}=\left(\begin{array}{c}
\frac{u^{2}+v^{2}}{a} \\
0 \\
0
\end{array}\right), \\
\boldsymbol{\beta}=\left(\begin{array}{c}
0 \\
\frac{g}{a} \frac{\partial h}{\partial \hat{\theta}} \\
\frac{g}{a \cos \theta} \frac{\partial h}{\partial \lambda}
\end{array}\right),
\end{gathered}
$$

and

$$
\boldsymbol{\delta}=\left(\begin{array}{c}
0 \\
f u \\
-f v
\end{array}\right)
$$

If we define $\mathrm{V}=(X, Y, Z)^{T}$ as the velocity in ('artesian coordinates $(x, y, z)$ then

$$
\mathbf{v}_{s}=\mathbf{Q V}
$$

where

$$
\mathbf{Q}=\left(\begin{array}{ccc}
\cos \theta \cos \lambda & \cos \theta \sin \lambda & \sin \theta \\
-\sin \theta \cos \lambda & -\sin \theta \sin \lambda & \cos \theta \\
-\sin \lambda & \cos \lambda & 0
\end{array}\right)
$$

Substituting ( 62 ) into (5T) and multiplying by $\mathbf{Q}^{T}$ we obtain the Cartesian form

$$
\frac{\partial \mathrm{V}}{\partial t}+\mathbf{C V}+\mathbf{Q}^{T}(\alpha+\beta+\delta)=0
$$

In this equation

$$
\mathbf{C}=\mathbf{Q}^{T} \mathbf{S Q}=\left(\begin{array}{ccc}
\frac{\partial X}{\partial x} & \frac{\partial X}{\partial y^{\prime}} & \frac{\partial X}{\partial z} \\
\frac{\partial y}{\partial x} & \frac{\partial y}{\partial y} & \frac{\partial y}{\partial z} \\
\frac{\partial Z}{\partial x} & \frac{\partial Z}{\partial y} & \frac{\partial z}{\partial z}
\end{array}\right)
$$




$$
\begin{gathered}
\mathbf{Q}^{T} \boldsymbol{\alpha}=\frac{1}{a^{2}}\left(\begin{array}{c}
x\left(X^{2}+Y^{2}+Z^{2}\right) \\
y\left(X^{2}+Y^{2}+Z^{2}\right) \\
z\left(X^{2}+Y^{2}+Z^{2}\right)
\end{array}\right), \\
\mathbf{Q}^{T} \boldsymbol{\delta}=\frac{2 \Omega z}{a^{2}}\left(\begin{array}{ccc}
0 & -z & y \\
z & 0 & -x \\
-y & x & 0
\end{array}\right)\left(\begin{array}{l}
X \\
Y \\
Z
\end{array}\right),
\end{gathered}
$$

and

$$
\mathbf{Q}^{T} \boldsymbol{\beta}=\mathbf{P} \nabla_{c} h
$$

where

$$
\mathbf{P}=\frac{g}{a^{2}}\left(\begin{array}{ccc}
a^{2}-x^{2} & -x y & -x z \\
-x y & a^{2}-y^{2} & -y z \\
-x z & -y z & a^{2}-z^{2}
\end{array}\right),
$$

and

$$
\Gamma_{c} h=\left(\frac{\partial h}{\partial x}, \frac{\partial h}{\partial y}, \frac{\partial h}{\partial z}\right)^{T}
$$

Similarly the continuity equation in Cartesian form is

$$
\frac{\partial h^{*}}{\partial t}+\mathbf{V}^{T} \mathbf{P V} \nabla_{c} h^{*}+h^{*} \nabla_{c} \cdot \mathbf{V}=0
$$

The matrix $P$ projects an arbitrary Cartesian vector onto a plane that is tangent to the sphere at the point $(x, y, z)$. For methods of evaluating the $\mathbf{C}$ matrix and the Cartesian gradient the reader is referred to [24].

\section{Test Cases}

The following test cases are proposed to evaluate and compare numerical schemes intended for global atmospheric models. The series increases in complexity. We suggest the tests be run in order without proceeding to the next until the numerical scheme is reasonably successful on the curlent one. For some schemes some of the requested parameter settings define trivial test and realistically provide only a superficial check of the code rather than a useful measure of the quality of the scheme. These situations should be jedentified so that no false condusions are drawn. Ideally the full set should be reported for each proposed scheme and trivial ases for that scheme acknowledged.

('ase 2 also provides a benchuark for timing implementations on various machines. It exercises the complete set of efpations and since it is a steady state solution no extra computations are required during the integration. For timing purposes an integration should be performed with all extra output processes removed after it has been demonstrated that the schene and codes solve the problem properly.

These tests represent necessary conditions only, i.e. any scheme must do well in these tests compared to currently acceptable schemes. Any scheme that performs well in these testos can then be incorporated in a global baroclinic general circulation model with state-of-the-alt physics and definitive tests can be condected. 
Parameters relevant to the earth and all test cases are

$$
\begin{aligned}
a & =6.37122 \times 10^{6} \mathrm{~m} \\
\Omega & =7.292 \times 10^{-5} \mathrm{~s}^{-1} \\
g & =9.80616 \mathrm{~m} \mathrm{~s}^{-2} .
\end{aligned}
$$

Unless specifically mentioned, the height of the mountains is taken to be zero $\left(h_{s}=0\right)$ and $h^{*}=h$.

\subsection{Advection of Cosine Bell over the Pole}

This is the only case of the suite that does not deal with the complete shallow water $e_{4}$ uations. It tests the advective component in isolation. Many shallow water codes can be easily changed for this test by overwriting the predicted wind field every time step with the analytically specified advecting wind. Since this wind field is nondivergent the equation for the height of the free surface reduces to the advection equation. For some methods, semi-implicit for example, some additional changes may be required to isolate the height forecast from the wind forecast.

A cosine bell is advected once around the sphere. Several orientations of the advecting wind are specified including around the equator, directly over the poles and minor shifts from these two orientations to avoid symmetries. This case is specified in equs. (4.2)-(4.5) of Williamson and Rasch [31]. The advecting wind is given by

$$
\begin{gathered}
u=u_{0}(\cos \theta \cos \alpha+\sin \theta \cos \lambda \sin \alpha) \\
v=-u_{0} \sin \lambda \sin \alpha .
\end{gathered}
$$

In terms of strean function and velocity potential this is

$$
\begin{gathered}
\psi^{\prime}=-a u_{0}(\sin \theta \cos \alpha-\cos \lambda \cos \theta \sin \alpha) \\
1=0 .
\end{gathered}
$$

The parameter $\alpha$ is the angle between the axis of solid body rotation and the polar axis of the spherical coordinate system. Tests should be run with $\alpha=0.0,0.05, \pi / 2-0.05$ and $\pi / 2$.

The initial cosine bell test pattern that is to be advected is given by

$$
h(\lambda . H)= \begin{cases}\left(h_{0} / 2\right)\left(1+\cos \frac{\pi r}{R}\right) & \text { if } r<R \\ 0 & \text { if } r \geq R\end{cases}
$$

where $h_{0}=1000 \mathrm{~m}$ and $r$ is the great circle distance between $(\lambda, \theta)$ and the center, initially taken as $\left(\lambda_{c}, \theta_{c}\right)=\left(\frac{3 \pi}{2}, 0\right)$.

$$
r=a \arccos \left[\sin \theta_{c} \cdot \sin \theta+\cos \theta_{c} \cdot \cos \theta \cos \left(\lambda-\lambda_{c}\right)\right] .
$$

The radius $R=\frac{n}{3}$ and the advectiag wind velocity $u_{0}=2 \pi a /(12$ days $)$, which is 
equivalent to about $40 \mathrm{~m} / \mathrm{sec}$. This solution translates without any change of shape.

Error measurcs: Plots of contour lines (interval $=100 \mathrm{~m}$ with zero contour) on orthographic projection with perspective centered over the true solution. True solution should also be contoured on the same plot with dashes but without the zero contour. Plot after one rotation. Contour maps of the error should also be provided after one rotation.

Some global measures of the error are also desirable. Define $I$ to be a discrete approximation to the global integral

$$
I(h)=\int_{0}^{2 \pi} \int_{-\frac{\pi}{2}}^{\frac{\pi}{2}} h(\lambda, \theta) \cos \theta d \theta d \lambda,
$$

which is consistent with the numerical approximations being tested; for example, Gaussian quadrature would be selected for the spectral transform method. The following normalized global errors should be graphed as a function of time sampled each time step where $h_{T}$ is the true solution.

$$
\begin{aligned}
\ell_{1}(h) & =I\left[\left|h(\lambda, h)-h_{T}(\lambda, \theta)\right|\right] / I\left[\left|h_{T}(\lambda, \theta)\right|\right] \\
\ell_{2}(h) & =\left\{I\left[\left(h(\lambda, \theta)-h_{T}(\lambda, \theta)\right)^{2}\right]\right\}^{\frac{1}{2}} /\left\{I\left[h_{T}(\lambda, \theta)^{2}\right]\right\}^{\frac{1}{2}} \\
\ell_{\infty}(h) & =\max _{\text {all. } \theta}\left|h(\lambda, \theta)-h_{T}(\lambda, \theta)\right| / \max _{\text {alL }, \theta}\left|h_{T}(\lambda, \theta)\right| .
\end{aligned}
$$

In addition, the normalized mean, variance, minimum and maximum values shouid be graphed is a function of time sampled each time step. Let $\bar{h}$ denote the mean

$$
h=I[h(\lambda, \theta)] / 4 \pi,
$$

then the normalized mean and variance are written

$$
\begin{aligned}
M & =\left(h-\ddot{h}_{T}\right) / \vec{h}_{0} \\
\nu & =\left\{I\left[(h-h)^{2}\right]-I\left[\left(h_{T}-\ddot{h}_{T}\right)^{2}\right]\right\} / I\left[\left(h_{0}-\ddot{h}_{0}\right)^{2}\right]
\end{aligned}
$$

and the minimum and maximum

$$
\begin{aligned}
& \left.h_{\text {max }}=\operatorname{madx}_{\substack{\text { mall, }, \theta \\
\text { and }}} h(\lambda, \theta)-\max _{\text {all } 1, \theta} h_{T}(\lambda, \theta)\right) / \Delta h \\
& h_{\min }=\left(\min _{a|l| l, t} h(\lambda . \theta)-\operatorname{minh}_{\text {all } 1 ., \theta} h_{T}(\lambda, \theta)\right) / \Delta h
\end{aligned}
$$

where $J h$ is the difference between the maximum and minimum values of the true solution intially and $h_{T}$ and $h_{0}$ are the true solution and intial field respectively.

\subsection{Global Steady State Nonlinear Zonal Geostrophic Flow}

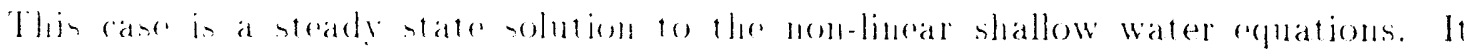
consint of eolid body rotation of zonal How with the corresponding geostrophic height 
field. The Coriolis parameter is a function of latitude and longitude so the flow can be specified with the spherical coordinate poles not necessarily coincident with earth's rotation axis. Again several orientations are specified.

The velocity field from egns. (4.1) (4.2) of Williamson and Browning [29] is initially (and for all time)

$$
\begin{gathered}
u=u_{0}(\cos \theta \cos \alpha+\cos \lambda \sin \theta \sin \alpha) \\
v=-u_{0} \sin \lambda \sin \alpha .
\end{gathered}
$$

In this report tia angle $\alpha$ has the opposite sign as that in Williamson and Browning [29] but the same sign as that in Williamson and Rasch [31]. In terms of stream function and velocity potential, the velocity field is

$$
\begin{gathered}
\psi=-a u_{0}(\sin \theta \cos \alpha-\cos \lambda \cos \theta \sin \alpha) \\
\downarrow=0 .
\end{gathered}
$$

The absolute vorticity is

$$
\eta=\left(\frac{2 u_{0}}{a}+2 \Omega\right)(-\cos \lambda \cos \theta \sin \alpha+\sin \theta \cos \alpha) .
$$

The analytic $h$ field is given by

$$
g h=g h_{0}-\left(a \Omega u_{0}+\frac{u_{0}^{2}}{2}\right)(-\cos \lambda \cos \theta \sin x+\sin \theta \cos \alpha)^{2} .
$$

It may be desirable to modify the initial wind and height fields so they satisfy a discrete nonlinear geostrophic relationship consistent with the scheme being tested. This could prevent spurious gravity waves from contaminating the numerical solution. The discrete balance may also be used to define the true solution for the purposes of calculating the error diagnostics. These changes are allowed but must be reported with the results along with the error comparing the discrete initial state to the analytic. The Coriolis paraneter associated with this solution is

$$
f=2 \operatorname{se} 2-\cos \lambda \cos \theta \sin \alpha+\sin \theta \cos \alpha) .
$$

The parameter values used should be $\|_{0}=2 \pi a / 12$ days $)$ as in case 1 and $g h_{0}=$ $2.94 \times 10^{4} \mathrm{m1}^{2} / \mathrm{s}^{2}$.

Tests should be run with $a=0.0,0.05, \pi / 2-0.0 .5$, and $\pi / 2$.

Error measures: ('ontour maps on an orthographice projection centered on $(3 \pi / 2, \pi / 4)$ of $h$ field and error after five days. Ciraph of the $\ell_{1}, l_{2}$, and $\ell_{x}$ errors of $h$ and $v$ versus time. The herrors are computed as in (\$2) - (\$.1). The v errors are given by

$$
C_{1}(\mathbf{v})=\frac{\left.I\left[\{u(\lambda . \theta)-u T(\lambda \cdot \theta))^{2}+(v(\lambda, \theta)-v(\lambda, \theta))^{2}\right\}^{\frac{1}{2}}\right]}{I\left[\left\{u(\lambda \cdot \theta)^{2}+\omega(\lambda, \theta)^{2}\right\}^{\frac{1}{2}}\right]}
$$




$$
\begin{aligned}
\ell_{2}(\mathbf{v}) & =\frac{\left\{I\left[\left(u(\lambda, \theta)-u_{T}(\lambda, \theta)\right)^{2}+\left(v(\lambda, \theta)-v_{T}(\lambda, \theta)\right)^{2}\right]\right\}^{\frac{1}{2}}}{\left\{I\left[u_{T}(\lambda, \theta)^{2}+v_{T}(\lambda, \theta)^{2}\right]\right\}^{\frac{1}{2}}} \\
\ell_{\infty}(\mathbf{v}) & =\frac{\max _{\text {all }, \theta}\left[\left\{\left(u(\lambda, \theta)-u_{T}(\lambda, \theta)\right)^{2}+\left(v(\lambda, \theta)-v_{T}(\lambda, \theta)\right)^{2}\right\}^{\frac{1}{2}}\right]}{\max _{\text {all } \lambda, \theta}\left[\left\{u_{T}(\lambda, \theta)^{2}+v_{T}(\lambda, \theta)^{2}\right\}^{\frac{1}{2}}\right]}
\end{aligned}
$$

In addition to these graphs a mesh convergence study should be performed. The $\ell_{2}(h)$ and $\ell_{2}(\mathbf{v})$ errors at five days for three different resolutions should be shown and a rate of convergence for the method estimated.

\subsection{Steady State Nonlinear Zonal Geostrophic Flow with Compact Support}

This case is similar to the previous except the wind field is nonzero in a limited region. It was introduced by Browning et al. [3]. In the editorial process for that paper some terms were dropped from the last equation in the first column on page 1068. It should read

$$
\begin{aligned}
\tilde{v} & =u(\cos \alpha \cos \tilde{\lambda} \sin \lambda \sin \tilde{\theta} \\
& -\cos \lambda \sin \tilde{\lambda} \sin \tilde{\theta}+\sin \alpha \sin \lambda \cos \tilde{\theta}) .
\end{aligned}
$$

This case is easiest to write first in a coordinate system $\left(\lambda^{\prime}, \theta^{\prime}\right)$ whose poles are concident with the Eanth's rotation axis, followed by a rotation through an angle $\alpha$ to the system $(\lambda, \theta)$ in which the jet is not parallel to the coordinate lines. This is essentially the process used to derive the equations above for solid body rotation, however, in the case with compact support it is more difficult to write the equations in closed form in the $(\lambda, \theta)$ system. Therefore, we present the equations in a series of steps. The velocities components $\left(u^{\prime}, v^{\prime}\right)$ in the $\left(\lambda^{\prime}, \theta^{\prime}\right)$ system are given by

$$
\begin{aligned}
& u^{\prime}=u_{o} b(x) b\left(x_{\epsilon}-x\right) e^{4 / x_{e}} \\
& v^{\prime}=0
\end{aligned}
$$

where

$$
b(x)= \begin{cases}0 . & x \leq 0 \\ e^{-x^{-1}}, & 0<x\end{cases}
$$

and

$$
x=x_{c}\left(\theta^{\prime}-\theta_{b}\right)\left(\theta_{c}-\theta_{b}\right)^{-1} .
$$

The parameters are $u_{0}=2 \pi a /\left(12\right.$ days). $\theta_{b}=-\pi / 6, \theta_{\epsilon}=\pi / 2$ and $x_{t}=0.3$. Note that $u^{\prime}$ is infinitely differentiable and has compact support. The stream function and velocity potential are given by

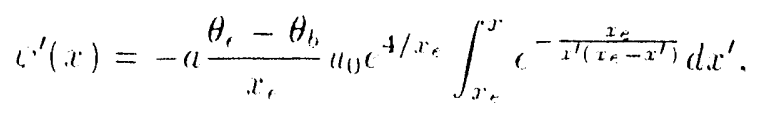

and

$$
1^{\prime}=0
$$


Following (5.13) to (5.17) of [31] (with $\lambda_{A}=0$ and $\theta_{A}=\alpha$ ) the rotated form can be written

$$
\begin{aligned}
v \cos \theta & =-u^{\prime} \sin \alpha \sin \lambda^{\prime} \\
u \cos \lambda & =v \sin \theta \sin \lambda+u^{\prime} \cos \lambda^{\prime}
\end{aligned}
$$

with the coordinates related by

$$
\begin{aligned}
\sin \theta^{\prime} & =\sin \theta \cos \alpha-\cos \theta \cos \lambda \sin \alpha \\
\sin \lambda^{\prime} \cos \theta^{\prime} & =\sin \lambda \cos \theta .
\end{aligned}
$$

The quadrant in which $\lambda^{\prime}$ falls can be determined by insuring that

$$
\sin \theta=\sin \theta^{\prime} \cos \alpha+\cos \theta^{\prime} \sin \alpha \cos \lambda^{\prime}
$$

is also satisfied. Equation (110) may suffer from precision problems because of the nesting of trigonometric and inverse trigonometric functions. A more stable test is that the principal value $\left(\lambda_{p}^{\prime}\right)$ is used for $\lambda^{\prime}$ when

$$
\cos \alpha \cos \lambda \cos \theta+\sin \alpha \sin \theta \geq 0
$$

otherwise $\lambda^{\prime}=\pi-\lambda_{p}^{\prime}$. This relationship can be obtained by transforming to Cartesian coordinates, rotating the Cartesian coordinates and noting that the principal value is needed in the primed system when $x^{\prime} \geq 0$. (The $x$ and $z$ coordinates are chosen to go through $(\lambda, \theta)=(0,0)$ and $\left(0, \frac{\pi}{2}\right)$, respectively, and the $y$ coordinate can be ignored.) The Coriolis parameter in the two systems is

$$
\begin{aligned}
& f=2 \Omega \sin \theta^{\prime} \\
& f=2 \Omega(-\cos \lambda \cos \theta \sin \alpha+\sin \theta \cos \alpha) .
\end{aligned}
$$

For a steady state solution $h^{\prime}$ must satisfy

$$
\frac{\left(u^{\prime}\right)^{2} \tan \theta^{\prime}}{a}+\frac{g}{a} \frac{\partial h^{\prime}}{\partial \theta^{\prime}}+f u^{\prime}=0 .
$$

For the general case the height is difficult to obtain antlytically. Therefore, we integrate the form in the prime system

$$
h=h_{0}-\frac{a}{g} \int_{-\frac{\pi}{2}}^{\theta^{\prime}}\left(2 \Omega \sin \tau+\frac{u^{\prime}(\tau) \tan \tau}{a}\right) u^{\prime}(\tau) d \tau
$$

numerically to obtain a highly accurate $h$. The background height, $h_{0}$, is given by $g / h_{0}=2.94 \times 10^{4} \mathrm{~m}^{2} / \mathrm{s}^{2}$ as in (ase 2 and the limit $\theta^{\prime}$ is related to $(\lambda, \theta)$, the point at which the geopotential is desired, by $(10 x)$.

Tests should be run with $a=0.0$, and $\pi / 3$.

Error measures: ('ontour maps of field and crror after five days on an orthographic: projection centered on $(3 \pi / 2 . \pi / 4)$. Ciraphs of the $\ell_{1}, \ell_{2}$ and $\ell_{\infty}$ errors of $h$ and $\mathbf{v}$ 
as functions of time. In addition to these graphs a mesh convergence study should be performed. The $\ell_{2}(h)$ and $\ell_{2}(\mathbf{v})$ errors at five days for three different resolutions should be shown for the $\alpha=\frac{\pi}{3}$ case and a rate of convergence for the method estimated.

\subsection{Forced Nonlinear System with a Translating Low}

The nonlinear steady state tests presented in the previous sections are the simplest measure of the adequacy of a particular numerical method. The performance a scheme on the nonlinear unsteady equations is also desirable, but analytic solutions are all but nonexistent. Thus, we take the approach followed by Browning et al. [3] who choose a flow $\tilde{u}, \tilde{v}$, and $\tilde{h}$ that is similar in structure to flows observed in the atmosphere. This flow is a solution to the forced shallow water system which can be written in advective form as

$$
\begin{gathered}
\frac{d u}{d t}-\frac{u v \tan \theta}{a}+\frac{g}{a \cos \theta} \frac{\partial h}{\partial \lambda}-f v=F_{u}, \\
\frac{d v}{d t}+\frac{u u \tan \theta}{a}+\frac{g}{a} \frac{\partial h}{\partial \theta}+f u=F_{v}, \\
\frac{d h}{d t}+\frac{h}{a \cos \theta}\left[\frac{\partial u}{\partial \lambda}+\frac{\partial v \cos \theta}{\partial \theta}\right]=F_{h},
\end{gathered}
$$

where the height of the mountains $h_{s}$ is taken to be zero and the substantial derivative is defined as

$$
\frac{d}{d t}=\frac{\partial}{\partial t}+\frac{u}{a \cos \theta} \frac{\partial}{\partial \lambda}+\frac{v}{a} \frac{\partial}{\partial \theta}
$$

and the forcing terms are defined as

$$
\begin{gathered}
F_{u}=\frac{d \tilde{u}}{d t}-\frac{\tilde{u} \tilde{v} \tan \theta}{a}+\frac{g}{a \cos \theta} \frac{\partial \tilde{h}}{\partial \lambda}-f \tilde{v} \\
F_{v}=\frac{d \tilde{v}}{d t}+\frac{\tilde{u} \tilde{u} \tan \theta}{a}+\frac{g}{a} \frac{\partial \tilde{h}}{\partial \theta}+f \tilde{u} \\
F_{h}=\frac{d \tilde{h}}{d t}+\frac{\tilde{h}}{a \cos \theta}\left[\frac{\partial \tilde{u}}{\partial \lambda}+\frac{\partial \tilde{v} \cos \theta}{\partial \theta}\right] .
\end{gathered}
$$

The flow is given by

$$
\begin{aligned}
\ddot{u} & =u-\frac{\dot{\phi} \theta}{a} \\
i & =\frac{\phi}{a \cos \theta} \\
g i & =g h+f \iota,
\end{aligned}
$$

whese glh $=1 h_{0}^{5} \mathrm{~m}^{2} / \mathrm{s}^{2} \cdot f_{0}=2 \Omega 2 \sin \frac{\pi}{1}$ and

$$
u=u_{0} \sin ^{1 \cdot 1}(2 \theta) \text {. }
$$




$$
\begin{gathered}
g h=g h_{0}-\int_{-\frac{\pi}{2}}^{\theta}[a f(\tau)+\ddot{u}(\tau) \tan \tau] \bar{u}(\tau) d \tau, \\
\bar{\psi}(\lambda, \theta, t)=\psi_{0} e^{-\sigma \frac{1-C}{1+C}}
\end{gathered}
$$

wi。 - $\psi_{0}=-0.03 \frac{g h_{0}}{f_{0}}, \sigma=(12.74244)^{2}$ and

$$
C=\sin \theta_{0} \sin \theta+\cos \theta_{0} \cos \theta \cos \left(\lambda-\frac{u_{0}}{a} t-\lambda_{0}\right)
$$

The center of the low is initially located at $\left(\lambda_{0}, \theta_{0}\right)=\left(-\frac{7 \pi}{12}, \frac{\pi}{4}\right)$. The velocity potential, $\chi$, is zero while the stream function is given by

$$
\psi(\theta, \lambda, t)=-\int_{-\frac{\pi}{2}}^{\theta} a \bar{u}(\tau) d \tau+\bar{\psi}(\theta, \lambda, t)
$$

The flow is a translating low pressure center superimposed on a jet stream which is symmetrical about the equator. Figure 5 of [3] illustrates the initial height field. This field exhibits some of the properties of middle level tropospheric flow (i.e., a short-wave trough embedded in a westerly jet).

The analytic expressions for the forcing are presented above for momentum. Schemes predicting other variables such as vorticity/divergence or stream function/velocity potential must be able to accept the forcing in terms of momentum as that is what is provided from the parameterizations in atmospheric models (see for example [30].) Thus solutions should be provided using the momentum forcing as prescribed. However, for the purpose of comparison with other schemes it may be advantageous to specify the forcing analytically in terms of the predicted variables if other than momentum. This approach is also allowed for these tests, but if it is chosen, then results with momentum forcing should also be presented.

Tests should be run with $u_{0}=20$ and $40 \mathrm{~m} / \mathrm{s}$.

Error measures: Contour maps of solution and error after 5 days on an orthographic projection centered on $\left(\lambda_{c}, \pi / 4\right)$, where $\lambda_{c}$ is the longitude of the center of the cell. The $\ell_{1}, \ell_{2}, \ell_{\infty}$ errors of $h^{\prime}$ and $\mathbf{v}^{\prime}$ should be plotted as a function of time. Here $h^{\prime}$ and $\mathbf{v}^{\prime}$ are the perturbation fields obtained by subtracting the background zonal flow

$$
\begin{aligned}
& h^{\prime}=h-h \\
& u^{\prime}=u-u \\
& u^{\prime}=v
\end{aligned}
$$

where $u$ and $h$ are given by $(126)$ and $(127)$ respectively. The true solution is modified in the sane way for the error calculation. The mean zonal component is removed so that the error prindrily represents that associated with the cell. The graphs should include data sampled every time step so that any oscillatory behavior can be seen. 


\subsection{Zonal Flow Over an Isolated Mountain}

This case was used by Takacs to study the effect of a posteriori methods for conservation of integral invariants [25]. It consists of zonal flow as in case 2 impinging on a mountain. The wind and height field are as in case 2 , with $\alpha=0$, but the mean height is changed to $h_{0}=5400 \mathrm{~m}$. The surface or mountain height is given by

$$
h_{s}=h_{s_{0}}(1-r / R)
$$

where $h_{s_{0}}=2000 \mathrm{~m}, R=\pi / 9$ and $r^{2}=\min \left[R^{2},\left(\lambda-\lambda_{c}\right)^{2}+\left(\theta-\theta_{c}\right)^{2}\right]$.

The center is taken as $\lambda_{c}=-\pi / 2$ and $\theta_{c}=\pi / 6$. As no analytical solution is known, a reference sciution will be provided by a high resolution spectral transform model integration. This will be provided as spectral coefficients at 5-day intervals and a routine to generate point values at arbitrary points. Agreement must be found with at least one other high resolution solution provided by a different numerical scheme in order to have confidence in the error measures.

Error masures: Contour maps on a rectangular latitude/longitude projection $(\Delta \dot{\lambda} / \Delta x=\Delta \theta / \Delta y)$ of the $h$ field and error at days 5, 10 and 15. Graphs of the $\ell_{1}, \ell_{2}$ and $\ell_{\infty}$, errors of $h$ and $\mathbf{v}$ calculated versus the high resolution solution plotted as a function of time sampled daily.

\subsection{Rossby-Haurwitz Wave}

Rossby-Haurwitz waves are analytic solutions of the nonlinear barotropic vorticity equation on the sphere [9]. Although they are not analytic solutions of the shallow water equations they have been used so frequently for meteorological tests that since Phillips' [19] first tests they have become de facto standard test cases although generally with different parameters selected by each investigator.

The initial velocity field is nondivergent and given by the streain function,

$$
\psi=-a^{2} \omega \sin \theta+a^{2} K^{2} \cos ^{R} \theta \sin \theta \cos R \lambda
$$

where $\omega . K$ and $R$ are constants. Haurwitz [9] showed that this pattern moves from west to east without change of shape in a nondivergent barotropic model with angular velocity $\nu$ given by

$$
\nu=\frac{R(3+R) \omega-2 \Omega}{(1+R)(2+R)}
$$

The velocity components are given by

$$
\begin{aligned}
& u=a \omega \cos \theta+a K \cos ^{R-1} \theta\left(R \sin ^{2} \theta-\cos ^{2} \theta\right) \cos R \lambda \\
& v=-a R R \cos ^{R-1} \theta \sin \theta \sin R \lambda
\end{aligned}
$$

and the vorticity by

$$
\zeta=2 \omega \sin \theta-K \sin \theta \cos ^{R} \theta\left(R^{2}+3 R+2\right) \cos R \lambda .
$$

The height is obtained from the stream function by solving the balance equation so the 
initial tendency of the divergence is zero [19].

$$
\begin{aligned}
g h= & g h_{0}+a^{2} A(\theta)+a^{2} B(\theta) \cos R \lambda+a^{2} C(\theta) \cos (2 R \lambda) \\
A(\theta)= & \frac{\omega}{2}(2 \Omega+\omega) \cos ^{2} \theta+\frac{1}{4} K^{2} \cos ^{2} \theta\left[(R+1) \cos ^{2} \theta\right. \\
& \left.+\left(2 P^{2}-R-2\right)-2 R^{2} \cos ^{-2} \theta\right] \\
B(\theta)= & \frac{2(\Omega+\omega) K}{(R+1)(R+2)} \cos ^{R} \theta\left[\left(R^{2}+2 R+2\right)\right. \\
& \left.-(R+1)^{2} \cos ^{2} \theta\right] \\
C(\theta)= & \frac{1}{4} K^{2} \cos ^{2 R} \theta\left[(R+1) \cos ^{2} \theta-(R+2)\right] .
\end{aligned}
$$

In the past the qualitative aspects of the solutions have generally been examined. To compliment the qualitative aspects we provide a reference solution from a high resolution spectral transform model integration. This will be provided as daily spectral coefficients and a routine to generate point values at an arbitrary point. The parameters are $\omega=h^{\circ}=7.848 \times 10^{-6} \mathrm{sec}^{-1}$ and $h_{0}=8 \times 10^{3} \mathrm{~m}$. Only a wave number 4 is chosen for the initial condition. Unstable waves [11] are not chosen since slightly different perturbations may lead to growth of different unstable modes as might be indicated in Kreiss and Oliger [12].

Error measures: Contour maps on a rectangular latitude/longitude projection $(\Delta \lambda / \Delta x=\Delta \theta / \Delta y)$ of the $h$ field and error at day 0,7 and 14 . The $\ell_{1}, \ell_{2}, \ell_{\infty}$ errors of $h$ and $\mathbf{v}$ calculated versus the high resolution solution plotted as a function of time sampled daily. Various normalized global invariants of the continuous equations should also be plotted as a function of time. Define the normalized integral

$$
I_{i}(\psi(t))=\{I[\psi(\lambda, \theta, t)]-I[\psi(\lambda, \theta, 0)]\} / I[\psi(\lambda, \theta, 0)]
$$

where the discrete integral operator $I$ is defined as (81). The following invariants should be presented:

geopotential $(i=1)$

$$
\psi=g h
$$

total energy $(i=2)$

$$
\vartheta=h(g h+\mathbf{v} \cdot \mathbf{v} / 2),
$$

vorticity $(i=3$ )

$$
\varphi^{\prime}=\zeta .
$$

divergence $(i=4)$

$$
\psi=\delta
$$


and potential enstrophy $(i=5)$

$$
\psi=0.5(\zeta+f)^{2} /(g h)
$$

\subsection{Analyzed $500 \mathrm{mb}$ Height and Wind Field Initial Conditions}

The last case consists of atmospheric initial conditions of the $500 \mathrm{mb}$ height and winds from several atmospheric states. The first is for 0000 GMT 21 December 1978, which Ritchie [21] used to test his semi-Lagrangian scheme. This case, with strong flow over the North Pole, has pointed out shortcomings of schemes in the past. A second case is 0000 GMT 16 January 1979 . This case is characterized initially by two cut-off lows. The flow pattern develops into a typical blocking situation. It has been studied extensively by Bengtsson [2]. The third case is 0000 GMT 9 January 1979, which initially has strong zonal flow. The last two cases are from the FGGE case studies selected by WGNE and discussed by Baumhefner and Bettge [1]. The shallow water equations should not necessarily be expected to predict the atmosphere well in these cases. The variety is chosen to illustrate any variability in the characteristics of schemes depending on atmospheric state.

In all cases the initial data are truncated to T 42 spectral resolution, which includes all scales resolved by the analyses. Ideally, nonlinear normal mode initialization consistent with the scheme being tested should be applied to the initial data to prevent gravity waves from contaminating the solution. The changes made by the initialization scheme should be submitted along with the error summary. However, because of the extra work necessary to develop the initialization codes, an initialized data set is also provided which is obtained via nonlinear normal mode initialization with a high resolution spectral transform model. Althrugh it may be advantageous to use an initialization procedure consistent with the scheme being tested, the choice is left to the scheme's proponents.

Error measures: The 'true' or reference solution will be obtained initially with the spectral transformation method applied to the finest resolution possible. Agreement must be found between at least two different schemes at high resolution to have confidence. The reference solution will be provided in terms of spherical harmonic coefficients so that it can be reconstituted on any computational grid. The $\ell_{1}, \ell_{2}$ and $\ell_{\infty}$ errors of $h$ and $\mathbf{v}$ should be plotted daily from 5-day forecasts. In addition, plots on north and south polar stereographic projections of the forecast and forecast error should be provided for day 1 and day 5 . The five global invariants listed with the Rossby-Haurwitz wave (Case 6) should also be graphed as a function of time.

\section{Performance Benchmark}

To exhibit the performance of a numerical scheme on a given computer system, the computer ('PU time and storage requirements for a 5 -day run of case 2 with $\alpha=\pi / 4$ (to avoid most symmetries) should be reported for various resolutions. The number of time steps taken and the errors in $h$ and $v$ at 5 days, as in (82)-(84) and (97)-(99) should be given for each resolution. Any time step restrictions or special cases should 
be recorded so that the computational effort corresponding to a climate simulation can be judged. Enough data should be provided so that comparisons can be made between schemes based on the computational resources required to achieve a given level of accuracy. These should include the total CPC time required, the number of operations required for the calculation, a measure of the sustained computational rate in gigaflops. and the data space storage required for each resolution. The machine and the compiler used should also be documented.

For parallel computers the wall clock time, as measured on the host computer. should be reported as well as the maximum time spent on any one processor. The maximum size of the data space required on any processor should also be reported. Execution times for a given resolution with the use of increasing numbers of processors. should be given to indicate how the algorithm scales. The speedup and parallel efficiency for each resolution should be given as a function of the number of processors. The parallel speedup is defined as $S_{p}=T_{1} / T_{p}$. where $T_{1}$ is the time required to execute the sequential algorithm on a single processor and $T_{p}$ is the execution time for the parallel algorithm using $p$ processors. The parallel efficiency is given by $E_{p}=S_{p} / p$. These measures may require an approximation of $T_{1}$ due to memory constraints in the single processor case. The method and assumptions used to approximate $T_{1}$ should be clearly stated. Co output or unnecessary computation should be performed during the j-day simulation.

\section{Genera! Comments}

Ideally all contouring should be via linear interpolation on the original computational grids without smoothing or additional interpolation to an intermediate grid in order to provide an indication of any noise in the solution. The utility of the rarious tests included in this suite will become apparent as more investigators apply their schemes to them. We hope investigators will use all the tests and publish in refereed journat selected results that illustrate both the strengths and weaknesses of the schemes. In-house technical reports containing the results from all the tests could provide the complete documentation of a scheme. We expect the suite will evolve informally with time 's investigators pont out weaknesses in the tests and suggest alternatives with arguments as to why they are good test cases. Several other cases are currently under consideration for inclusion. These consist of Thompson's nonlinear series solution to the equations [26] and modons in spherical geometry [27]. The latter do not have an a alvicical solution for the shallow water equations and a high resolution numerical sulution will be required for a reference solution. J. (ote (personal communication)

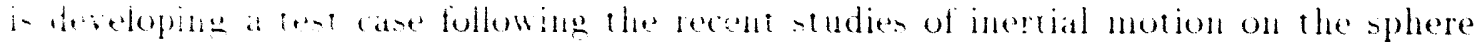
16 . It. Thi care will complement the pure advection (ane 1 and deal only with the momentum equations.

The test suite will only becones tandard to the extent the communty finds it

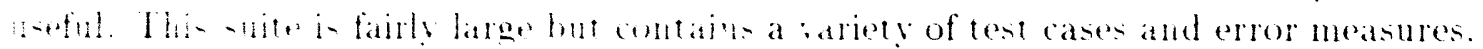

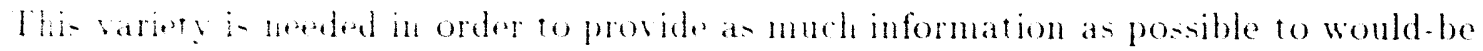

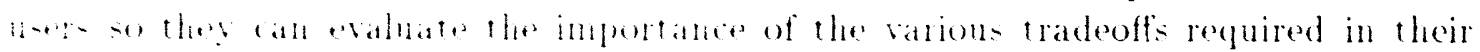
aplitiotions 


\section{References}

[1] D. P. Baumhefner and T. W. Bettge. Characteristics of atmospheric planetary circulation and associated model forecast skill during FGGE case studies selected by WGNE. In report of International Conference on Early Results of FGGE and Large-Scale Aspects of Its Monsoon Experiments, pages 2.26-2.31. WMO, Geneva, 1981.

[2] L. Bengtsson. Numerical prediction of atmospheric blocking-A case study. Tellus. $33: 19-42,1981$.

[3] G. L. Browning, J. J. Hack, and P. N. Swarztrauber. A comparison of three numerical methods for solving differential equations on the sphere. Mon. Wea. Rev., 117:1058-1075, 1989.

[4] J. Coté. A Lagrange multiplier approach for the metric terms of semi-Lagrangian models on the sphere. Quart. J. R. Met. Soc., 114:1347-1352, 1988.

[5] J. Cóté and A. Staniforth. A two-time-level semi-Lagrangian semi-implicit scheme for spectral models. Mon. Wea. Rev., 116:2003-2012, 1988.

[6] ECMWF. Techniques for hoizontal discretization in numerical weather prediction models. Proceedings of a workshop held at ECMWF, 2-4 November 1987, European Centre fo: Medium-Range Weather Forecasts, 1988.

[i] E. Eliasen. B. Machenhauer, and E. Rasmussen. On a numerical method for integration of the hydrodynamical equations with a spectral representation of the horizontal fields. Technical Report Report No. 2, Institut for teoretisk meteorologi, Eniversity of Copenhagen, Copenhagen, 1970.

[8] G. J. Haltiner and R. T. Williams. Numerical Prediction and Dynamic Meteorology. John Wiley and Sons. New York, second edition, 1980.

[9] B. Haurwitz. The motion of atmospheric disturbances on the spherical earth. $J$. of Marine Res.. 3:25+4 26i. 1940.

[10] J. J. Holton. An Introduction to Dynamic Meteorology. Academic Press, New rork. second edition. 1979.

(11] B. J. Hoskins. Stability of the Rossby-Haurwitz wave. Quart. J. R. Met. Soc.. 99:7-2:3 7.45. 197:3.

[12] H.-O. Kreiso and J. Oliger. Comparison of accurate methods for the integration of hiperbolic equations. Tellus, 2.4:199 215, 1972.

[13] B. Machenhaver. The spectral method. In Vumerical Methods Vsed in Atmosphe ric Models, chapter 3, pages 121-275. (iARP Pub. Ser. No. 17. JOC, WMO. Geneva. Switzerland. 1979. 
[14] Y. Masuda and H. Ohnishi. An integration scheme of the primitive equation model with an icosahedral-hexagonal grid system and its application to the shallow water equations. In Short- and Medium-Range Numerical Weather Prediction, pages $317-326,1986$.

[15] S. A. Orszag. Transform method for calculation of vector-coupled sums: Application to the spectral form of the vorticity equation. J. Atmos. Sci., 27:890-895, 1970 .

[16] N. Paldor and P. D. Killworth. Inertial trajectories on a rotating earth. J. Atmos. Sci., 45:4013-4019, 1988.

[17] F. Pearson. Map Projections: Theory and Applications. CRC Press, Boca Raton, Florida, second edition, 1990.

[18] S. A. Pennell and K. L. Seitter. On inertial motion on a rotating sphere. J. Atmos. Sci, $47: 2032-2034,1990$.

[19] N. A. Phillips. Numerical integration of the primitive equations on the hemisphere. Mon. Hea. Rev. 8i:333-345, 1959.

[20] P. J. Rasch and D. L. Williamson. Sensitivity of a general circulation model climate to the moisture transport formulation. J. Geophys. Res., accepted, 1991.

[21] H. Ritchie. Application of the semi-Lagrangian method to a spectral model of the shallow water equations. Mon. Wea. Rev., 116:1587-1598, 1988.

[2:] W. J. Saucier. Principles of Meteorological Analysis. The University of Chicago Press. Chicago, 1962.

[2:3] J. A. Steers. An Introduction to the Study of Map Projections. University of London Press, Ltd. London, 1962.

[2-4] P. N. Swarztrauber. The approximation of vector functions and their derivatives on the sphere. SIAM J. Numer. Anal. 18:191 210. 1981.

[25] L. L. Takacs. Effects of using a posteriori methods for the conservation of integral invariants. Hon. Hia Ror.. 116:525)-545. 1988.

[26] P. D. Thompson. Haurwitz solutions of the nonlineas shallow-water equations for

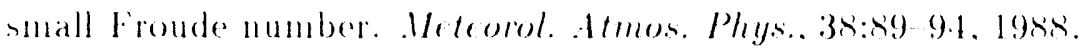

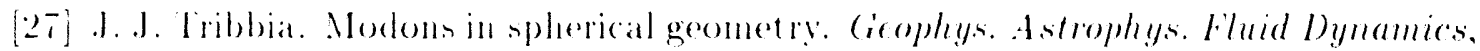
$301: 131 \quad 16 \times 2.19 \times 1$.

[2- D] D. L. Williamson. Difference approximations for fluid flow on a sphere. In Numerical Me thods lised in Atmospherie Models. chapter 2. pages 51 120. (iARP Pub. Ser. No. 17.JO('. WMO. Ceneva. Switzerland. 1979. 
[29] D. L. Williamson and G. L. Browning. Comparison of grids and difference approximations for numerical weather prediction over a sphere. J. Appl. Meteor., $12: 264-274,1973$.

[30] D. L. Williamson, J. T. Kiehl, V. Ramanathan, R. E. Dickinson, and J. J. Hack. Description of NCAR Community Climate Model (CCM1). NCAR Tech. Note NCAR/TN-285+STR, National Center for Atmospheric Research, Boulder, Colo., 1987 .

[31] D. L. Williamson and P. J. Rasch. Two-dimensional semi-Lagrangian transport with shape-preserving interpolation. Mon. Wea. Rev., 117:102-129, 1989. 
ORNL/TM-11895

\section{INTERNAL DISTRIBUTION}

1. B. R. Appleton

2-3. T. S. Darland

4. J. J. Dongarra

5. T. H. Dunigan

6-10. J. B. Drake

11. W. R. Emanuel

12. R. E. Flanery

13-17. S. A. Raby

18-22. R. F. Sincovec

23. D. W. Walker

\author{
24-28. R. C. Ward \\ 29-33. P. H. Worley \\ 34. Central Research Library \\ 35. ORNL Patent Office \\ 36. K-25 Applied Technology \\ Library \\ 37. Y-12 Technical Library \\ 38. Laboratory Records - RC \\ 39-40. Laboratory Records Department
}

\section{EXTERNAL DISTRIBUTION}

41. Christopher R. Anderson, Department of Mathematics, University of California, Los Angeles, CA 90024

42. David C. Bader, Atmospheric and Climate Research Division, Office of Health and Environmental Research, Offic of Energy Research, ER-76, U.S. Department of Energy, Washington, DC 20585

43. David H. Bailey, NASA Ames, Mail Stop 258-5, NASA Ames Research Center, Moffet Ficld, CA 94035

44. Edward H. Barsis, Computer Science and Mathematics, P. O. Box 5800, Sandia National Lahoratory, Albuquerque, NM 87185

45. Colin Bennett, Department of Mathematics, University of South Carolina, Columbia, SC. 29208

46. Dominicgue Bronet1. ('FRFAC'S. 42 Avenue (iustave Coriolis, 31057 Toulouse (inlex. FRANCF

17. Narsha J Berger. ('ourant hustitute of Mathematical Siciences. 251 Mercer Street, Niw Yink NY 101112

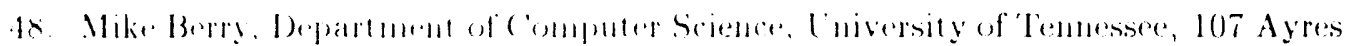

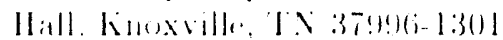

19. Ake Bjorck. Departument of Mathematics. Linkoping Liniversity, S-58 83 Linkoping. Siverden

50. Jolun H. Bohatad Lawrence Lirermore National Laboratory. L-16, P. O. Box 808,

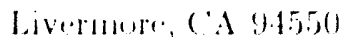

51. (iecorge Bromianoff. Supercondurting Super (odlider Laboratory, 2550 Beckleymeade

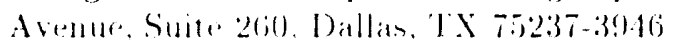

52. Roger II. Brockett, Wang Professor of EE and (S, Division of Applied Sciences, Harvard Enversity ('ambrilge. 11402138 
53. Bill L. Buzbee, Scientific Computing Division, National Center for Atmospheric Research, P.O. Box 3000, Boulder, CO 80307

54. Captain Edward A. Carmona, Parallel Computing Research Group, U. S. Air Force Weapons Laboratory, Kirtland AFB, NM 87117

55. Peter Campbell, Environmental Science Divsion, Argonne National Laboratory, 9700 South Cass Avenue, Argonne, IL 60439

56. John Cavallini, Acting Director, Scientific Computing Staff, Applied Mathematical Sciences, Office of Energy Research, U.S. Department of Energy, Washington, DC 20585

57. I-liang Chern, Mathematics and Computer Science Division, Argonne National Laboratory, 9700 South Cass A venue, Argonne, IL 60439

58. Ray Cline, Sandia National Laboratories, Livermore, CA 945う்0

59. Alexandre Chorin, Mathematics Department, Lawrence Berkeley Laboratory, Berkeley, CA 94720

60. Jannes C'orones, Ames Lahoratory, lowa State University, Ames, IA 50011

61. Jean Coté, RPN, 2121 'Transcanada Highway, Suite 508, Dorval, Quebec H9P 1J3, CANADA

62. William Dannevik, Lawrence Livermore National Laboratory, P. O. Box 808, L-16, Livermore, CA 94550

63. John J. Dorning, Department of Nuclear Engineering Physics, Thornton Hall, McCormick Road, University of Virginia, Charlottesville, VA 22901

64. Iain S. Duff, Atlas Centre, Rutherford Appleton Laboratory, Didcot, Oxon OX11 OQX, England

65. John Dukowicz, Los Alamos National Laboratory, Group T-3, Los Alamos, NM 87545

66. Richard E. Ewing, Department of Mathematics, University of Wyoming, Laramie, WY 82071

67. Ian Foster, Mathematics and Computer Science Division, Argonne National Laboratory, 9700 South Cass Avenue, Argonne, IL 60439

68. Geoffrey C. Fox, NPAC, 111 College Place, Syracuse University, Syracuse, NY $13244-4100$

69. Paul O. Frederickson, RIACS, MS 230-5, NASA Ames Research Center, Moffet Field. ( id 94035

70. J. Alan (ieorge, Vice President, Academic and Provost, Needles Hall, University of Waterloo, Waterloo, Ontario. (ANADA N2L 3G1

71. Janes Glimm, Department of Mathematics, State University of New York, Stony Brook, NY 11794

72. Gene Golub, Computer Science Department, Stanford University, Stanford, CA 94305

73. Johu Gustafson, 236 Milleelu, Ames Laboratory, lowa State University, Ames, IA 50011 
74. Phil Gresho, Lawrence Livermore National Laboratory, L-262, P. O. Box 808, Livermore, CA 94550

75. William D. Gropp, Mathematics and Computer Science Division, Argonne National Laboratory, 9700 South Cass Avenue, Argonne, IL 60439

76. Eric Grosse, AT\&'T Bell Labs 2T-504, Murray Hill, NJ 07974

77. James J. Hack, National Center for Atmospheric Research, P. O. Box 3000, Boulder, $\mathrm{CO}$ óv307

78. Michael T. Heath, Center for Supercomputing Research and Development, 305 Talbot Laboratory, University of Illinois, 104 South Wright Street, Urbana, IL $61801-2932$

79. Michael Henderson, Los Alamos National Laboratory, Group T-3, Los Alamos, NM 87545

80. Lennar Johnsson, Thinking Machines Inc., 245 First Street, Cambridge, MA $02142-1214$

81. J.R. Jump, ECE Dept., Rice University, P.O. Box 1892, Houston, 'TX 77251

82. Malvyn Kalos, Comell Theory Center, Engineering and Theory Center Bldg., Cornell University, Ithaca, NY 14853-3901

83. Hans Kaper, Mathematics and Computer Science Division, Argonne National Laboratory, 9700 South Cass Avenue, Argonne, IL 60439

84. Alan H. Karp, IBM Scientific Center, 1530 Page Mill Road, Palo Alto, CA 94304

85. Kenneth Kennedy, Department of Computer Science, Rice University, P. O. Box 1892, Houston, Texas 77001

86. Tom Kitchens, ER- 7, Applied Mathematical Sciences, Scientific Computing Staff, Oflice of Energy Research, Office (i-437 (Germantown, Washington, D(: 2() 585

87. Peter D. Lax, Courant Institute of Mathematical Sciences, New York University, 251 Mercer Street, New York, NY 10012

88 . Janes L. Leiss, Rt. 2. Box 1126 Broadway, VA 22\% is

89. Rich Loft, National (enter for Amospheric Research, P. O. Box 3000, Boulder, (.) 803037

90). Michael ( Mackacken, Lawrence Livenume National Laboratory, L-262, P. O. Box $8(0)$, livermore, ('A 9.550

91. Norman D). Malmuth, Science ('enter, Rockwell International Corporation, 1049 (amino Dos Rios, P.(). Box 1085, Thousand Oaks, (A 91358

92. Robert Matone, Los Alamos National Laboratory, (-3, Mail Stop B265, Los Alamos, NM 87545

93. Len Margolin, Los Alamos National Laboratory, Los Alamos, NM 87545

94. Frank Mce 'abe. Departunent of (omputing, Inperial College of Science and Technology, Iso Queens (iate. London SWT 2BZ, ENGLAND)

95. Jantes Meciraw, Lawrence Livernore National Laboratory, L-306, P. O. Box 808, Livermore. (A 94550 
96. L. David Meeker, Mathematics Department, University of New Hampshire, Kingsbury Hall, Durham, NH 03824

97. Paul C. Messina, Mail Code 158-79, Califoiaia Institute of Technology, $1201 \mathrm{E}$. California Blvd. Pasadena, CA 91125

98. Neville Moray, Department of Mechanical and Industrial Engineering, University of Illinois, 1206 West Green Street, Urbana, IL 61801

99. V. E. Oberacker, Department of Physics, Vanderbilt University, Box 1807, Station B, Nashville, TN 37235

100. Joseph Oliger, Computer Science Department, Stanford University, Stanford, CA 94305

101. Robert O'Malley, Department of Mathematical Sciences, Rensselaer Polytechnic Institute, Troy, NY 12180-3590

102. James M. Ortega, Department of Applied Mathematics, Thornton Hall, University of Virginia, Charlottesville, VA 22901

103. Ron Peierls, Applied Mathematical Department, Brookhaven National Laboratory, Upton, NY 11973

104. Richard Pelz, Dept. of Mechanical and Aerospace Engineering, Rutgers University, Piscataway, NJ 08855-0909

105. Paul Pierce, Intel Scientific Computers, 15201 N.W. Greenbrier Parkway, Beaverton, OR 97006

106. Robert J. Plemmons, Departments of Mathematics and Computer Science, North Carolina State University. Raleigh, NC 27650

107. Jesse Poore, (omputer Science Department, University of Tennessee, Knoxville, T'N $37996-1300$

108. Andrew Priestley, Institute for ('omputational Fluid Dynamics, Reading University, Reading RGi6 2AX, EN(ILAND

109. Lee Riedinger, Director. The Science Alliance Program, University of Tennessee, knoxville, TN 37996

110. Garry Rocirigue. Numerical Mathematics Group, Lawrence Livermore National Laboratory, Livermore, CA 94550

111. Ahmed Sameh, Department of Computer Science, 200 Union Street, S.E., University of Minnesota, Minneapolis, MN 554555

112. Dave Schneider, University of Illinois at Urbana-Champaign, Center for Supercomputing Research and Development, 319E Talbot - 104 S. Wright Street Urbana, IL. 61801

113. David S. Scott, Intel Scientific Computers, 15201 N.W. Greenbrier Parkway, Beaverton, OR 97006

114. William C. Skanarock, 3973 Escuela ('ourt, Boulder, CO 80301

115. Richard Smith, Los Alanos National Laboratory, Group T-3, Mail Stop B2316, Los Alamos. NM $\times 7545$

116. Peter Smolarkiewic\% National ('enter for Atmospheric Research, MMM Ciroup,

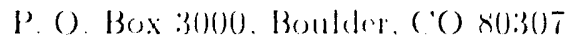


117. Jurgen Steppeler, DWD, Frankfurterstr 135, 6050 Offenbach, WEST GERMANY

118. Rick Stevens, Mathematics and Computer Science Division, Argonne National Laboratory, 9700 South Cass Avenue, Argonne, IL 60439

119. Paul N. Swarztrauber, National Center for Atmospheric Research, P. O. Box 3000, Boulder, CO 80307

120. Wei Pai Tang, Department of Computer Science, University of Waterloo, Waterloo, Ontario, Canada N2L 3G1

121. Harold Trease, Los Alamos National Laboratory, Mail Stop B257, Los Alamos, NM 87545

122. Roiert G. Voigt, ICASE, MS 132-C, NASA Langley Research Center, Hampton, VA 23665

123. Mary F. Wheeler, Rice University, Department of Mathematical Sciences, P. O. Box 1892, Houston, TX 77251

124. Andrew B. White, Los Alamos National Laboratory, P. O. Box 1663, MS-265, Los Alamos, NM 87545

125. David L. Williamson, National Center for Atmospheric Research, P. O. Box 3000, Boulder, (O 8030T

126. Samuel Yee, Air Force Geophysics Lab, Department LYP, Hancom AFB, Bedford, MA 01731

127. Office of Assistant Manager for Energy Research and Development, U.S. DepartInent of Energy, Oak Ridge Operations Office, P. O. Box 2001, Oak Ridge, TN $37831-8600$

128-137. Office of Scientific \& Technical Information, P. O. Box 62, Oak Ridge, TN 37831 

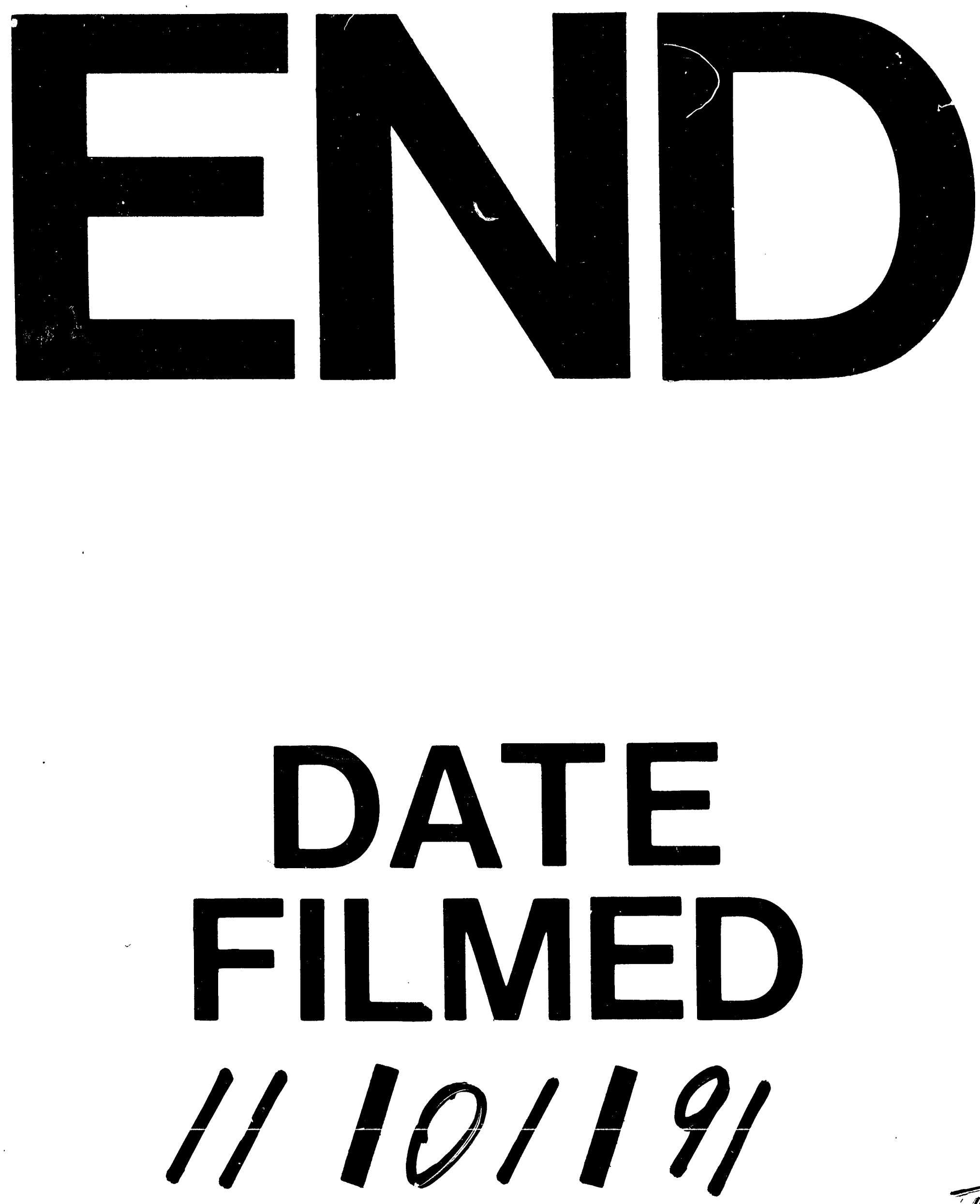
